\title{
Review of the Pathophysiology, Diagnosis, and Orthopedic Treatment Options for Juvenile Idiopathic Arthritis
}

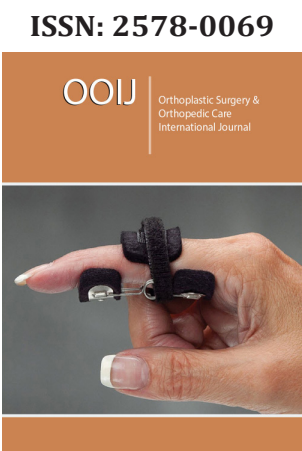

*Corresponding author: Phong Truong, Department of Orthopedic Surgery, Larkin Community Hospital, 7000 SW 62nd Ave, Suite 401, South Miami, FL 33143, USA

Submission: 泟 January 05, 2021

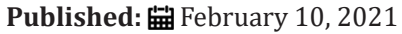

Volume 2 - Issue 2

How to cite this article: Phong Truong, Lori Chambers, Cindy Ho, Cameron McLaury, Margaret Kell. Review of the Pathophysiology, Diagnosis, and Orthopedic Treatment Options for Juvenile Idiopathic Arthritis. Ortho Surg Ortho Care Int J . 2(2). 00IJ.000535. 2021. DOI: 10.31031/OOIJ.2021.02.000535

Copyright@ Phong Truong, This article is distributed under the terms of the Creative Commons Attribution 4.0 International License, which permits unrestricted use and redistribution provided that the original author and source are credited.

\author{
Phong Truong ${ }^{1 *}$, Lori Chambers ${ }^{2}$, Cindy $\mathrm{Ho}^{1}$, Cameron Mc Laury ${ }^{3}$ and Margaret \\ Kell ${ }^{4}$ \\ ${ }^{1}$ Department of Orthopedic Surgery, Larkin Community Hospital, USA \\ ${ }^{2}$ Department of General Surgery, Upstate Medical University, USA \\ ${ }^{3}$ College of Medicine, Oklahoma University Health Sciences Center, USA \\ ${ }^{4}$ New York Institute of Technology, Arkansas State University, USA
}

\begin{abstract}
While juvenile idiopathic arthritis is the most common inflammatory joint condition within the pediatric population, the burden of this pathology can be lessened by early detection and interdisciplinary management to avoid severe joint damage and skeletal deformity. The etiology of juvenile idiopathic arthritis is largely unknown; however, immunologic studies show possible involvement of CD141+, CD123+, and dendritic cells. The natural disease course consists of inflammatory soft tissue damage coupled with joint effusion that eventually progresses to bone and joint changes. In terms of diagnosis, musculoskeletal ultrasonography has been shown to be effective in the early detection of juvenile idiopathic arthritis, especially in small joints. Magnetic resonance imaging and radiography are also valid techniques for diagnosis, but they generally fail to detect preclinical juvenile idiopathic arthritis, whereas ultrasound is successful in doing so. Orthopedic treatment options include conservative measures such as non-steroidal inflammatory drugs and corticosteroid injections. Surgical intervention is often indicated to treat deformity, limb length, and severe arthritis. Total joint replacement is primarily performed for functional impairment and deformity rather than for pain. Factors that may complicate surgical intervention include small bone size, limb deformity, and soft tissue contracture.
\end{abstract}

Keywords: Juvenile idiopathic arthritis; Juvenile rheumatoid arthritis; Pediatric; Orthopedic surgery; Total knee arthroplasty; Total hip arthroplasty

Abbreviations: JIA: Juvenile Idiopathic Arthritis; RA: Rheumatoid Arthritis; SA: Septic Arthritis; MSUS: Musculoskeletal Ultrasonography; MRI: Magnetic Resonance Imaging; THA: Total Hip Arthroplasty; TKA: Total Knee Arthroplasty; TSA: Total Shoulder Arthroplasty; ILAR: International League of Associations for Rheumatology

\section{Introduction}

Juvenile idiopathic arthritis (JIA) is the most common inflammatory joint disease in children [1]. it is described as joint inflammation of unknown origin lasting longer than six weeks [2,3]. Since chronic arthritis varies between race and location, it is important to analyze the frequency of JIA in a diverse population of patients. In the United States, JIA has an estimated incidence of 11.9 cases per 100,000 children and a prevalence of 44.7 cases per 100,000 children. The occurrence of JIA is most common in Caucasian children and is significantly less common in African-American, Asian, and Hispanic children [4]. Like rheumatoid arthritis (RA), JIA causes chronic joint pain and inflammation that can lead to physical or ocular disability [5]. An early interdisciplinary approach is necessary to improve outcomes and decrease disease activity, which may delay or avoid the need for eventual joint replacement [6]. Fortunately, the need for arthroplasty in JIA may be decreasing. One study evaluated the rates of arthroplasty in multiple conditions, including RA and JIA. More than 2.8 million knee, hip, and shoulder arthroplasties between 1991 and 2005 were analyzed in the study. In that timeframe, the rate of arthroplasty for RA slightly decreased from 4.6 per 100,000 to 4.5 per 100,000 , but the rate of arthroplasty for JIA decreased by nearly 50 percent-from 0.22 per 100,000 to 0.13 per 100,000 . In addition, the age at time 
of arthroplasty increased in the JIA patient-from 30.9 years to 36.7 years. The decreased rate of arthroplasty and increased age at time of arthroplasty is likely due to higher risk of fracture and prosthetic failure, growth and remodeling of bone, and higher complication rates in younger patients [7]. However, MertelsmannVoss et al. note that the timeline of this decline in operative rates and increased age of operation for JIA coincides with the advent of biologic agents, which are likely successful in preventing end-stage joint damage [8].

\section{Discussion}

\section{Pathophysiology of JIA}

Although the etiology and pathogenesis of JIA is still unknown the older literature suggests theories of exposures in the prenatal and infantile environments that would likely lead to triggering an early inflammatory reaction; however, more recent literature points to specific biomarkers and gastrointestinal microbiome involvement that may suggest innate immunity contributes to a larger role in the manifestation of JIA [1,9].

Several studies investigated the prenatal environment and found no association between birth weight, maternal age, maternal smoking, and the increased risk of JIA in their children. Breast milk was hypothesized to have a protective factor towards JIA [1]. Contrary to this hypothesis, Palman et al. found that breast fed children developed JIA at an earlier age with less severity compared to infants not given breast milk. Infants and their siblings are shown to have an association named the "hygiene hypothesis" in which the increase in siblings and earlier exposure to pathogens demonstrated a decreased likelihood of developing JIA $[10,11]$. New studies have investigated specific biomarkers that are involved in the first line of defense-the innate immune system. A prospective study compared immunological biomarkers in an attempt to find discriminatory signals for the initiation of JIA and septic arthritis (SA). Cren et al. found that in synovial fluid, CD141+, CD123+, and dendritic cells were found in higher numbers in JIA compared to SA [12]. These markers play a notable role in the overactivation of monocytes and dendritic cells in JIA and differentiate their pathophysiological mechanism compared to biomarkers that specifically activate the inflammatory response in SA [12].

The natural progression of this disease is known to follow a specific pattern of inflammatory soft tissue damage coupled with joint effusion that eventually progress to osseous changes. Depending on the severity of the immunological response, this can lead to a variety of manifestations. Current management falls short due to the limited knowledge of the biological processes of the disease. Biologic agents that target the immune system are expensive, require long-term treatment, and do not treat the disease definitively $[5,13]$. Eisenstein et al. explain that pediatric rheumatologists may need to provide a "more precise biological classification" to specify and improve treatments that target gene expression and new immunological findings that contribute to this disease process [14].

\section{Diagnosis of JIA}

To better facilitate the complexities of diagnosis and treating JIA, a pediatric rheumatologist is generally involved from initial stages. When formulating a differential diagnosis, severe diseases that are life threatening and need immediate intervention should be excluded first. The International League of Associations for Rheumatology (ILAR) compiled new nomenclature, definitions, and diagnostic criteria to replace the previous classification of "Juvenile Rheumatoid Arthritis." The current definition of JIA is a diagnosis of exclusion encompassing a group of disease subtypes that can be characterized by the onset of arthritis before the age of 16 and symptoms lasting 6 weeks [15]. The diseases are categorized into seven subtype groups: systemic arthritis, oligoarthritis (persistent or extended), polyarthritis rheumatoid factor (RF)-positive, polyarthritis RF-negative, enthesitis-related arthritis (ERA), psoriatic arthritis (PsA), and undifferentiated arthritis [16].

In current literature, musculoskeletal ultrasonography (MSUS), radiography, and magnetic resonance imaging (MRI) are the most commonly used imaging modalities for the diagnosis and clinical assessment of patients with JIA. "Ultrasonography is known to be the simpler, more cost effective option with less adverse effects compared to radiographs and MRI [17]. It is more useful to capture the early erosive changes in the pediatric skeleton due to their cartilaginous composition which can be a hindrance with the use of radiography. Ventura-Ríos et al. demonstrated that MSUS provides good reliability in the evaluation of basic lesions found in JIA; however, literature is lacking in regards to the validation and standardization of ultrasound for patients with JIA $[18,19]$. They suggested that subclinical synovitis in the smaller joints, specifically the hand, wrist, elbow, and ankle, are more apparent via ultrasound compared to larger joints, such as the hip and knee [18]. While further research needs to demonstrate the validity of this modality, the combination of the clinical assessment and use of MSUS can improve the accuracy and sensitivity in detecting the exact location of inflammation in the joint. Magni-Manzoni explained how this can be important for therapeutic decisions such as corticosteroid injections and monitoring the efficacy for biological treatments [19].

"Radiography has been a historical tool for the evaluation of arthritis-especially for JIA". For initial diagnosis, it provides a baseline for long-term radiographic assessment, but not necessarily for routine surveillance of the patient. Radiographs help with the exclusion of other differentials for joint pain and swelling. They also aid in finding late osseous complications, such as limb length discrepancy and physeal fusion, which may warrant surgical intervention. To date, there is only one validated scoring system for the hip: The Childhood Arthritis Radiographic Score of the Hip. In adults with arthritis, scoring systems along with the structural damage visualized on radiograph begin to assist in tailoring new therapies $[13,20]$. The scarcity of scoring systems for radiographs in the pediatric population primarily leads to physicians using their clinical assessment of joint function and disability over the use of 
imaging features to solely guide management.

MRI is the only modality that can assess bone marrow changes and soft-tissue changes. It is easy to misinterpret the soft-tissue and bone in younger populations. Children have a rich branching vascular network in the epiphyseal canals that can allow contrast agents to diffuse faster and linger within the cartilage and provide an effect of hypertrophy and synovial changes that the inexperienced interpreter may mistake for abnormal signals [13]. What could be a normal phenomenon for the growing child may be mistaken for severe disease. There are also a lack of standardized scoring systems involving MRI for patients with JIA. In addition to avoiding the use of ionizing radiation, this imaging modality could benefit from further reliability and reproducibility studies to help standardize imaging protocols and assist in the management of JIA [13].

\section{Orthopedic treatment options for JIA}

Orthopedic treatment for JIA involves both conservative as well as surgical options. Like other orthopedic problems, conservative modalities include nonsteroidal anti-inflammatory medication and corticosteroids [21,22]. Methotrexate is regarded as a second line treatment for JIA. It should also be noted that adalimumab, a fully human monoclonal anti-TNF antibody, has received FDA approval for the treatment of JRA. Slow-acting antirheumatic drugs (SAARDs) may be considered in children unresponsive to other non-surgical treatment options. (KIM 2010) Canakinumab and tocilizumab, which are monoclonal anti-IL-1 $\beta$ and anti-IL-6 antibodies respectively are FDA approved agents that have been shown to be efficacious against JIA [23-25]. Even with the adjunct of rheumatologic medication such as disease-modifying antirheumatic drugs, JIA may progress to advanced arthritis, causing pain and disability in those affected [21]. Orthopedic surgical treatment is often necessary for correcting deformity, limb length discrepancy, or severe arthritis [2]. Operative methods include releasing of soft tissues, osteotomies, synovectomies, total joint replacements, and joint fusions [2,15,21]. Complex joint deformity caused by soft tissue contracture may complicate and decrease functionality. In order to correct this deformity, soft tissue release may be performed at the affected joints. In the shoulder and hip joint, extensive soft tissue release may be adequate for deformity correction. However, in the knee joint, soft tissue release often requires adjunct bony resection [15].

Total hip and knee replacement is often indicated for joint destruction, functional impairment, deformity, or severe pain [26]. "More commonly, joint replacement is performed for functional impairment and deformity-and less commonly for pain". When there are indications for both hip and knee arthroplasty in the same patient, one article suggests hip arthroplasty ought to be performed first [26]. One article states that 60 percent of patients undergoing total knee arthroplasty (TKA) for JIA have had prior surgery for total hip arthroplasty (THA) [27].
Several factors may complicate operative treatment in patients with JIA. Small bone size, limb deformity, and soft tissue contracture make the surgeries more difficult $[15,26]$. For example, in a patient with severe hip flexion contracture, extensive soft tissue release that causes leg lengthening may cause a nerve palsy [26]. One article suggests it may be better to delay joint replacement for as long as possible, as the highest rates of loosening and complications occur in patients who had surgery at a younger age [15]. Moreover, not only is primary surgical treatment difficult in JIA patients, but revision joint arthroplasty is also technically demanding [7]. Due to the patient's small proportions and low bone quality, the early complication rates are relatively high, and the long-term survivorship is guarded [7].

THA, as mentioned above, is one surgical treatment option for JIA. "Again, it is performed for functional impairment more often than pain. In fact, only 13 percent of patients report severe pain at time of surgery [15]." Joint contracture and altered anatomy make the operation more challenging. For example, acetabular deficiencies caused by chronic subluxation of the femoral head may necessitate bone grafting [15]. Also, distorted anatomy of the proximal femur-excessive anteversion or small femoral neckmay require a custom implant [15]. Overall, the results of THA in JIA are good, and the most common complication is loosening of the cemented acetabular component, similar to the rheumatoid arthritis population [15].

TKA is another orthopedic surgical treatment option for JIA. TKA is used to improve ambulation and decrease pain. The knee is susceptible to a valgus, flexion, and external rotation deformity which can greatly influence the outcome of surgery [15]. Another deformity results from premature growth plate closure, leading to a trumpet-shaped distal femur with a wide coronal dimension and a narrow sagittal dimension. Posterior capsule and lateral releases are performed, and bony resection is often necessary [15]. In addition, a posterior stabilized implant is often utilized, but use of a constrained prosthesis is not uncommon due to the need for extensive soft tissue release and bony resection mentioned above, which may lead to instability [15]. Adolescents undergoing total knee arthroplasty tend to have good survivorship. One study reports up to 95 percent survivorship at ten years [28].

Other joint replacement considerations include the shoulder, elbow, and ankle. Total shoulder arthroplasty (TSA) has proven to be more reliably used to treat pain, rather than restoration of motion, in patients with JIA. A custom prosthesis is often needed due to the small intramedullary canal of the humerus and smaller glenoid. Another important consideration is that an elbow replacement may also be necessary in the future, therefore it is crucial to use a short stemmed humeral component to allow room for the possible elbow replacement. Elbow replacement is reserved for severe elbow involvement, usually in the form of complete ankylosis, as the operation is considered to have an unacceptably high complication rate [15]. Likewise, ankle replacement for JIA is not considered 
in younger patients unless there is complete ankylosis, and often ankle arthrodesis is the recommended treatment option [15].

\section{Conclusion}

Since clinical symptoms of JIA can be quite variable, prompt diagnosis and treatment are essential in order to prevent longterm complications such as skeletal maturation impairment and damage to joints and organs. Conservative treatment modalities for JIA include nonsteroidal anti-inflammatory medication and corticosteroids. While conservative modalities are sufficient in some instances, JIA may still progress to advanced arthritis. In these cases, orthopedic surgical intervention is often necessary to correct deformity, limb length, and severe arthritis. Total joint replacement is primarily performed for functional impairment and deformity rather than for pain. Factors that may complicate surgical intervention include small bone size, limb deformity, and soft tissue contracture. Due to relatively high complication rates in the younger population, it may be best to delay joint replacement for as long as possible.

\section{References}

1. Palman J, Shoop Worrall S, Hyrich K, McDonagh JE (2018) Update on the epidemiology, risk factors and disease outcomes of Juvenile idiopathic arthritis. Best Pract Res Clin Rheumatol 32(2): 206-222.

2. Bovid KM, Moore MD (2019) Juvenile Idiopathic Arthritis for the Pediatric Orthopedic Surgeon. Orthop Clin North Am 50(4): 471-488.

3. Prakken B, Albani S, Martini A (2011) Juvenile idiopathic arthritis. Lancet 377(9783): 2138-2149.

4. Harrold LR, Salman C, Shoor S, Jeffrey RC, Maryam MA, et al. (2013) Incidence and prevalence of juvenile idiopathic arthritis among children in a managed care population, 1996-2009. J Rheumatol 40(7): 12181225.

5. Ailioaie LM, Litscher G (2020) Molecular and cellular mechanisms of arthritis in children and adults: New perspectives on applied photobiomodulation. Int J Mol Sci 21(18): 6565.

6. Arbogast M, Haas JP (2018) Treatment options in juvenile idiopathic arthritis: Part 2: Orthopedics und surgery. Orthopade 47(11): 919-927.

7. Goodman SB, Hwang K, Imrie S (2014) High complication rate in revision total hip arthroplasty in juvenile idiopathic arthritis. Clin Orthop Relat Res 472(2): 637-644.

8. Mertelsmann Voss C, Lyman S, Pan TJ, Goodman SM, Figgie MP, et al. (2014) US trends in rates of arthroplasty for inflammatory arthritis including rheumatoid arthritis, juvenile idiopathic arthritis, and spondyloarthritis. Arthritis Rheumatol 66(6): 1432-1439.

9. Giancane G, Alongi A, Ravelli A (2017) Update on the pathogenesis and treatment of juvenile idiopathic arthritis. Curr Opin Rheumatol 29(5): 523-529.

10. Okada H, Kuhn C, Feillet H, Bach JF (2010) The "hygiene hypothesis" for autoimmune and allergic diseases: An update. Clin Exp Immunol 160(1): $1-9$.
11. Bell SW, Shenoi S, Nelson JL, Bhatti P, Mueller BA (2017) Juvenile idiopathic arthritis in relation to perinatal and maternal characteristics: A case control study. Pediatr Rheumatol Online J 15(1): 36.

12. Cren M, Nziza N, Carbasse A, Perrine M, Emilie DL, et al. (2020) Differential Accumulation and Activation of Monocyte and Dendritic Cell Subsets in Inflamed Synovial Fluid Discriminates Between Juvenile Idiopathic Arthritis and Septic Arthritis. Front Immunol 11: 1716.

13. Shelmerdine SC, Di Paolo PL, Tanturri de HL, Malattia C, Magni MS, et al. (2018) Imaging of the hip in juvenile idiopathic arthritis. Pediatr Radiol 48(6): 811-817.

14. Eisenstein EM, Berkun Y (2014) Diagnosis and classification of Juvenile Idiopathic Arthritis. J Autoimmun 48(49): 31-33.

15. Kim HJ, Kahn B, Figgie MP (2008) Total joint replacement in childhood arthritis. Curr Rheumatol Rep 10(2): 135-141.

16. Martini A, Ravelli A, Avcin T, Michael WB, Ruben BV, et al. (2019) Toward new classification criteria for juvenile idiopathic arthritis: First steps, pediatric rheumatology international trials organization international consensus. J Rheumatol 46(2):190-197.

17. Rosendahl K, Maas M (2018) Update on imaging in juvenile idiopathic arthritis. Pediatr Radiol 48(6): 783-784.

18. Ventura-RL, Faugier E, Barzola L, LB De la CB, Guadalupe SB, et al. (2018) Reliability of ultrasonography to detect inflammatory lesions and structural damage in juvenile idiopathic arthritis. Pediatr Rheumatol 16(1): 58.

19. Magni MS (2016) Ultrasound in juvenile idiopathic arthritis. Pediatr Rheumatol 14(1): 33

20. Sheybani EF, Khanna G, White AJ, Demertzis JL (2013) Imaging of juvenile idiopathic arthritis: A multimodality approach. Radiographics 33(5):1253-1273.

21. Mitrogiannis L, Barbouti A, Theodorou E, Aikaterini K, Georgios M, et al. (2019) Surgical treatment of juvenile idiopathic arthritis: A review. Bull Hosp Joint Dis 77(2): 99-114.

22. Gandhi R, Mahomed N (2008) Juvenile Rheumatoid Arthritis and Total Hip Arthroplasty. Semin Arthroplasty 19(4): 261-266.

23. Lee JJY, Schneider R (2018) Systemic Juvenile Idiopathic Arthritis. Pediatr Clin North Am 65(4): 691-709.

24. Ruperto N, Brunner HI, Quartier P, Tamás C, Nico W, et al. (2012) Two Randomized Trials of Canakinumab in Systemic Juvenile Idiopathic Arthritis. N Engl J Med 367(25): 2396-2406.

25. Yokota S, Imagawa T, Mori M, Takako M, Yukoh A, et al. (2008) Efficacy and safety of tocilizumab in patients with systemic-onset juvenile idiopathic arthritis: a randomised, double-blind, placebo-controlled, withdrawal phase III trial. Lancet 371(9617): 998-1006.

26. Scott R, Sarokhan A, Dalziel R (1984) Total hip and total knee arthroplasty in juvenile rheumatoid arthritis. Clin Orthop Relat Res 182: 90-98.

27. Heyse TJ, Ries MD, Bellemans J, Stuart BG, Richard DS, et al. (2014) Total knee arthroplasty in patients with juvenile idiopathic arthritis. In: Clin Orthop Relat Res 472(1): 147-154.

28. Martin JR, Sutak AK, Milbrandt TA, Martin VA, Trousdale RT (2017) Adolescent total knee arthroplasty. Arthroplast Today 3(2):105-109. 